\title{
Global Existence and Uniform Energy Decay Rates for the Semilinear Parabolic Equation with a Memory Term and Mixed Boundary Condition
}

\author{
Zhong Bo Fang and Liru Qiu \\ School of Mathematical Sciences, Ocean University of China, Qingdao 266100, China \\ Correspondence should be addressed to Zhong Bo Fang; fangzb7777@hotmail.com
}

Received 14 July 2013; Accepted 18 September 2013

Academic Editor: Shaoyong Lai

Copyright ( 2013 Z. B. Fang and L. Qiu. This is an open access article distributed under the Creative Commons Attribution License, which permits unrestricted use, distribution, and reproduction in any medium, provided the original work is properly cited.

\begin{abstract}
This work is concerned with a mixed boundary value problem for the semilinear parabolic equation with a memory term and generalized Lewis functions which depends on both spacial variable and time. Under suitable conditions, we prove the existence and uniqueness of global solutions and the energy functional decaying exponentially or polynomially to zero as the time goes to infinity by introducing brief Lyapunov function and precise priori estimates.
\end{abstract}

\section{Introduction}

In this paper, we are concerned with the global existence and uniform energy decay rates for the nonlocal semilinear heat equation with a memory term and generalized Lewis function

$$
\begin{array}{r}
A(x, t) u_{t}-\Delta u+\int_{0}^{t} g(t-s) \operatorname{div}[a(x) \nabla u(s)] d s=0, \\
(x, t) \in \Omega \times(0, \infty),
\end{array}
$$

subjected to mixed boundary and initial conditions

$$
\begin{gathered}
-\frac{\partial u}{\partial v}+\int_{0}^{t} g(t-s)[a(x) \nabla u(s) \cdot v] d s=f(u), \\
(x, t) \in \Gamma_{0} \times(0, \infty), \\
u(x, t)=0, \quad(x, t) \in \Gamma_{1} \times[0, \infty), \\
u(x, 0)=u_{0}(x), \quad x \in \Omega,
\end{gathered}
$$

where $\Omega \subset \mathbb{R}^{n}(n \geq 1)$ is a bounded domain with sufficient smooth boundary $\partial \Omega$, such that $\partial \Omega=\Gamma_{0} \cup \Gamma_{1}, \Gamma_{0} \cap \Gamma_{1}=\emptyset$, and $\Gamma_{0}, \Gamma_{1}$ have positive measures, $\nu$ is the unit outward normal on $\partial \Omega, A(x, t)$ is a generalized Lewis function (when $A(x, t)=C$, $C$ is a positive constant, and $A(x, t)$ is called Lewis function; see [1]) which satisfies (i) positive function $A(x, t) \in W^{1, \infty}\left(0, \infty ; L^{\infty}(\Omega)\right)$ and $A_{t}(x, t) \leq 0$ a.e. for $t \geq 0$.

Equation (1) arises naturally from a variety of mathematical models in engineering and physical science. For example, in the study of heat conduction in materials with memory, the classical Fourier's law of heat flux is replaced by the following form:

$$
q=-d \nabla u-\int_{-\infty}^{t} \nabla[k(x, t) u(x, \tau)] d \tau,
$$

where $u, d$, and the integral term represent temperature, diffusion coefficient, and the effect of memory term in the material, respectively. The study of this type of equations has drawn a considerable attention; see [2-6]. From the mathematical point of view, one would expect the integral term in the equation to be dominated by the leading term. So the theory of parabolic equations can be applied to this type of equations.

Recently, many works were dedicated to studying the global existence, blow-up solutions, and asymptotic properties of the initial boundary value problem for the parabolic equation with memory term. In the absence of the memory 
term $(g \equiv 0)$, for the quasilinear parabolic equations with absorption term

$$
u_{t}=\operatorname{div}\left(|\nabla u|^{p-2} \nabla u\right)-f(u), \quad(x, t) \in \Omega \times(0, \infty),
$$

where $\Omega \subset \mathbb{R}^{n}(n \geq 1)$ is a bounded domain with smooth boundary and $p \geq 2$, there are many results about the global existence and finite time blow-up of solutions for the homogeneous Dirichlet boundary value problems; see [711]. The conclusions in Levine [7], Kalantarov, Ladyzhenskaya [8], and Levine et al. [9] showed that global and nonglobal existence depends on the nonlinearity of $f, p$, the dimension $n$, and the initial data. For the research on global existence and asymptotic properties of the solution, we refer the readers to $[10,11]$. Pucci and Serrin [10] studied the following equation with the homogeneous Dirichlet boundary conditions:

$$
A(t)\left|u_{t}\right|^{m-2} u_{t}=\Delta u-f(x, u), \quad(x, t) \in \Omega \times(0, \infty),
$$

where $m>1$ and the strong solution tends to 0 when $t \rightarrow$ $\infty$ under the condition $(f(x, u), u)>0$ but did not give the decay rate. Berrimi and Messaoudi [11] proved that if a bounded square matrix $A(t) \in C\left(\mathbb{R}^{+}\right)$satisfying

$$
(A(t) v, v) \geq c_{0}|v|^{2}, \quad t \in \mathbb{R}^{+}, v \in \mathbb{R}^{n},
$$

then the solution with small initial energy decays exponentially for $m=2$ and polynomially for $m>2$.

When there is a memory term $(g \neq \equiv \quad 0)$, Messaoudi [12] studied the semilinear heat equation with a power form source term

$$
\begin{array}{r}
u_{t}-\Delta u+\int_{0}^{t} g(t-s) \Delta u(x, s) d s=|u|^{p-2} u, \\
(x, t) \in \Omega \times(0, \infty),
\end{array}
$$

where the relaxation function $g: \mathbb{R}^{+} \rightarrow \mathbb{R}^{+}$is a bounded $C^{1}$ function and $p>2$; he proved the existence of blow-up solution with positive initial energy and the homogeneous Dirichlet boundary condition by convexity method. Later, Fang and Sun [13] improved the results of [12] with when $|u|^{p-2} u$ be replaced by fully nonlinear source term $f(u)$. For the study of general energy decay for the quasilinear parabolic system with a memory term, we see [14].

In the works mentioned above, there are few about the global existence and uniform energy decay rates of solution for parabolic equation with mixed boundary conditions. Motivated by it, we intend to study global existence and uniqueness of solutions for the mixed initial boundary value problem (1)-(2) with a memory term and generalized Lewis function by the Galerkin method and also give the estimates of uniform energy decay rates.

The main innovations of this paper are: (1) that the model is representative, considering the mixed boundary value problem with a generalized Lewis function and time integral boundary conditions, and $f, g$ are weak; (2) we give the reason and process of the definition of the energy functional; (3) we prove the energy decays exponentially or polynomially to zero as the time goes to infinity by introducing brief Lyapunov function and precise priori estimates.
The present work is organized as follows. In Section 2, we present the assumptions, lemmas, and energy functional for our work. In Section 3, we prove the existence and uniqueness of the global solution; Section 4 is devoted to proving the energy decay results.

\section{Preliminaries}

In the sequel we state the general hypotheses on the relaxation function $g$, coefficient $a$, nonlinearity $f$, and initial value $u_{0}$.

(H1) $g: \mathbb{R}^{+} \rightarrow \mathbb{R}^{+}, g(0)>0$ and $g$ is a nondecreasing differentiable function.

$(\mathrm{H} 2) a: \Omega \rightarrow \mathbb{R}^{+}$is a nonnegative bounded function and $a(x) \geq a_{0}>0$ with

$$
1-\|a\|_{L^{\infty}} \int_{0}^{\infty} g(s) d s=l>0 .
$$

(H3) The function $f: \mathbb{R} \rightarrow \mathbb{R}$ is Lipschitz continuous and satisfies

$$
f(s) s \geq 2 F(s) \geq 0, \quad s \in \mathbb{R},
$$

where $F(u):=\int_{0}^{u} f(s) d s$.

(H4) (Compatibility Condition) The initial value satisfies

$$
u_{0} \in V \cap H^{2}(\Omega), \quad-\frac{\partial u_{0}}{\partial \nu}=f\left(u_{0}\right)
$$

Remark 1. The condition $1-\|a\|_{L^{\infty}} \int_{0}^{\infty} g(s) d s=l>0$ is necessary to guarantee the parabolicity of the problem (1)-(2).

Throughout this paper, we define that

$$
V=\left\{u \mid u \in H^{1}(\Omega), u=0 \text { on } \Gamma_{1}\right\}
$$

and the following scalar products

$$
(u, v)=\int_{\Omega} u(x) v(x) d x, \quad(u, v)_{\Gamma_{0}}=\int_{\Gamma_{0}} u(x) v(x) d \Gamma,
$$

and norms

$$
\|u\|_{L^{p}(\Omega)}=\left(\int_{\Omega}|u|^{p} d x\right)^{1 / p}, \quad\|u\|_{L^{p}\left(\Gamma_{0}\right)}=\left(\int_{\Gamma_{0}}|u|^{p} d \Gamma\right)^{1 / p} .
$$

To simplify the notations, we denote $\|u\|_{L^{p}(\Omega)}$ and $\|u\|_{L^{p}\left(\Gamma_{0}\right)}$ by $\|u\|_{p}$ and $\|u\|_{p, \Gamma_{0}}$, respectively.

Next, we give some important lemmas which will be used in the proof of our main results. 
Lemma 2. For any $g, u \in C^{1}[0,+\infty)$, we have

$$
\begin{aligned}
\int_{\Omega} \int_{0}^{t} g(t-s) a(x) \nabla u(s) \nabla u^{\prime}(t) d s d x \\
=-\frac{1}{2} \int_{\Omega}\left[g(t)|\sqrt{a(x)} \nabla u(t)|^{2}-\left(g^{\prime} \circ \nabla u\right)\right] d x \\
-\frac{1}{2} \frac{d}{d t} \int_{\Omega}\left[(g \circ \nabla u)-\int_{0}^{t} g(s)\right. \\
\left.\times|\sqrt{a(x)} \nabla u(t)|^{2} d s\right] d x,
\end{aligned}
$$

where $(g \circ \nabla u)=\int_{0}^{t} g(t-s)|\sqrt{a(x)}(\nabla u(t)-\nabla u(s))|^{2} d s$.

Proof. Differentiating $\int_{\Omega}(g \circ \nabla u) d x$ with respect to $t$ and noting $\int_{0}^{t} g(t-s) d s=\int_{0}^{t} g(s) d s$ yield

$\frac{d}{d t} \int_{\Omega}(g \circ \nabla u) d x=\frac{d}{d t}$

$$
\begin{aligned}
& \times \int_{\Omega} \int_{0}^{t} g(t-s) \\
& \times \mid \sqrt{a(x)} \\
& \times\left.(\nabla u(t)-\nabla u(s))\right|^{2} d s d x \\
& =\int_{\Omega} \int_{0}^{t} g^{\prime}(t-s) \\
& \times|\sqrt{a(x)}(\nabla u(t)-\nabla u(s))|^{2} d s d x \\
& +2 \int_{\Omega} \int_{0}^{t} g(t-s) a(x) \\
& \times[(\nabla u(t)-\nabla u(s))] \\
& \times \nabla u^{\prime}(t) d s d x \\
& =\int_{\Omega}\left[g^{\prime}(t-s) \circ \nabla u\right] d x \\
& +2 \int_{\Omega} \int_{0}^{t} g(t-s) a(x) \nabla u(t) \\
& \times \nabla u^{\prime}(t) d s d x \\
& -2 \int_{\Omega} \int_{0}^{t} g(t-s) a(x) \nabla u(s) \\
& \times \nabla u^{\prime}(t) d s d x \\
& =\int_{\Omega}\left[g^{\prime}(t-s) \circ \nabla u\right] d x
\end{aligned}
$$

$$
\begin{gathered}
+\frac{d}{d t} \int_{\Omega} \int_{0}^{t} g(s)|\sqrt{a(x)} \nabla u(t)|^{2} d s d x \\
-\int_{\Omega} g(t)|\sqrt{a(x)} \nabla u(t)|^{2} d x \\
-2 \int_{\Omega} \int_{0}^{t} g(t-s) a(x) \nabla u(s) \\
\times \nabla u^{\prime}(t) d s d x,
\end{gathered}
$$

which implies

$$
\begin{gathered}
\int_{\Omega} \int_{0}^{t} g(t-s) a(x) \nabla u(s) \nabla u^{\prime}(t) d s d x \\
=-\frac{1}{2} \int_{\Omega}\left[g(t)|\sqrt{a(x)} \nabla u(t)|^{2}-\left(g^{\prime} \circ \nabla u\right)\right] d x \\
-\frac{1}{2} \frac{d}{d t} \int_{\Omega}\left[(g \circ \nabla u)-\int_{0}^{t} g(s)\right. \\
\left.\times|\sqrt{a(x)} \nabla u(t)|^{2} d s\right] d x .
\end{gathered}
$$

This completes the proof.

In order to define the energy functional $E(t)$ of the problem (1)-(2), we give the following computation. Multiplying (1) by $u_{t}$, integrating over $\Omega$, and using Green's formula, we get from Lemma 2 that

$$
\begin{aligned}
0= & \int_{\Omega} A(x, t) u_{t} u_{t} d x-\int_{\Omega} \Delta u u_{t} d x \\
& +\int_{\Omega} \int_{0}^{t} g(t-s) \operatorname{div}[a(x) \nabla u(s)] u_{t} d s d x \\
= & \int_{\Omega} A(x, t)\left|u_{t}\right|^{2} d x+\int_{\Omega} \nabla u \nabla u_{t} d x \\
& -\int_{\Omega} \int_{0}^{t} a(x) g(t-s) \nabla u(s) \nabla u^{\prime}(t) d s d x \\
& +\int_{\Gamma_{0}} f(u) u_{t} d \Gamma \\
= & \int_{\Omega} A(x, t)\left|u_{t}\right|^{2} d x \\
& +\frac{1}{2} \frac{d}{d t}\left(\int_{\Omega}|\nabla u|^{2} d x-\int_{\Omega} \int_{0}^{t} g(s) d s|\sqrt{a(x)} \nabla u(t)|^{2} d x\right. \\
& \left.+\int_{\Omega}(g \circ \nabla u) d x\right)+\frac{d}{d t} \int_{\Gamma_{0}} F(u) d \Gamma
\end{aligned}
$$




$$
\begin{aligned}
& +\frac{1}{2} \int_{\Omega}\left[g(t)|\sqrt{a(x)} \nabla u(t)|^{2}-\left(g^{\prime} \circ \nabla u\right)\right] d x \\
& =\frac{d}{d t}\left(\frac{1}{2} k(x, t) \int_{\Omega}|\nabla u|^{2} d x\right. \\
& \left.\quad+\frac{1}{2} \int_{\Omega}(g \circ \nabla u) d x+\int_{\Gamma_{0}} F(u) d \Gamma\right) \\
& +\int_{\Omega} A(x, t)\left|u_{t}\right|^{2} d x \\
& +\frac{1}{2} \int_{\Omega} g(t)|\sqrt{a(x)} \nabla u(t)|^{2} d x \\
& -\frac{1}{2} \int_{\Omega}\left(g^{\prime} \circ \nabla u\right) d x,
\end{aligned}
$$

where $k(x, t)=1-a(x) \int_{0}^{t} g(s) d s>1-\|a(x)\|_{L^{\infty}} \int_{0}^{\infty} g(s) d s>$ 0 .

The above computation inspires us to define the energy functional $E(t)$ of the problem (1)-(2) as

$$
\begin{aligned}
E(t)= & \frac{1}{2} k(x, t) \int_{\Omega}|\nabla u|^{2} d x \\
& +\frac{1}{2} \int_{\Omega}(g \circ \nabla u) d x+\int_{\Gamma_{0}} F(u) d \Gamma .
\end{aligned}
$$

We have the following properties about $E(t)$.

Lemma 3. The energy $E(t)$ is nonnegative and

$$
\begin{aligned}
\frac{d}{d t} E(t)= & \frac{1}{2} \int_{\Omega}\left[\left(g^{\prime} \circ \nabla u\right) d x-g(t)|\sqrt{a(x)} \nabla u(t)|^{2}\right] d x \\
& -A(x, t)\left|u_{t}\right|^{2} d x \leq 0 .
\end{aligned}
$$

To show the uniform decay of the solution, we introduce a functional

$$
\varphi(t)=\frac{1}{2} \int_{\Omega} A(x, t)|u(x, t)|^{2} d x .
$$

Here, we need to point out that $C$ denotes a positive constant not necessarily the same at different occurrences.

Lemma 4. There exists a positive constant $C$ such that

$$
|\varphi(t)| \leq C E(t), \quad t \geq 0 .
$$

Proof. By Poincaré inequality, we have

$$
\begin{aligned}
|\varphi(t)| & =\left.\left|\frac{1}{2} \int_{\Omega} A(x, t)\right| u(x, t)\right|^{2} d x \mid \\
& \leq \frac{\lambda C}{2} \int_{\Omega}|\nabla u|^{2} d x \leq C E(t),
\end{aligned}
$$

where $\lambda$ is a positive constant.
Lemma 5. There exist two positive constants $k_{1}$ and $k_{2}$, such that for some $T>0$, we have

$$
\frac{d}{d t} \varphi(t) \leq-k_{1} E(t)+k_{2} \int_{\Omega}(g \circ \nabla u) d x, \quad \forall t \geq T .
$$

Proof. Multiplying (1) by $u(t)$, integrating over $\Omega$, and using Green's formula, we get

$$
\begin{aligned}
0= & \int_{\Omega} A(x, t) u_{t} u(t) d x-\int_{\Omega} \Delta u(t) d x \\
& +\int_{\Omega} \int_{0}^{t} g(t-s) \operatorname{div}[a(x) \nabla u(s)] u(t) d s d x \\
= & \int_{\Omega} A(x, t) u_{t} u(t) d x+\int_{\Omega}|\nabla u|^{2} d x \\
& -\int_{\Omega} \int_{0}^{t} a(x) g(t-s) \nabla u(s) \nabla u(t) d s d x \\
& +\int_{\Gamma_{0}} f(u) u(t) d \Gamma .
\end{aligned}
$$

Differentiating $\varphi(t)$, we get

$$
\begin{aligned}
\frac{d}{d t} \varphi(t)= & \frac{1}{2} \int_{\Omega} A_{t}(x, t)|u(t)|^{2} d x \\
& +\int_{\Omega} A(x, t) u_{t} u(t) d x \\
= & \frac{1}{2} \int_{\Omega} A_{t}(x, t)|u(t)|^{2} d x-\int_{\Omega}|\nabla u|^{2} d x \\
& +\int_{\Omega} \int_{0}^{t} a(x) g(t-s) \nabla u(s) \nabla u(t) d s d x \\
& -\int_{\Gamma_{0}} f(u) u d \Gamma \\
= & \frac{1}{2} \int_{\Omega} A_{t}(x, t)|u(t)|^{2} d x-2 E(t) \\
& +k(x, t) \int_{\Omega}|\nabla u|^{2} d x+\int_{\Omega}(g \circ \nabla u) d x \\
& +2 \int_{\Gamma_{0}} F(u) d \Gamma-\int_{\Omega}|\nabla u|^{2} d x \\
& +\int_{\Omega} \int_{0}^{t} a(x) g(t-s) \nabla u(s) \nabla u(t) d s d x
\end{aligned}
$$




$$
\begin{aligned}
& -\int_{\Gamma_{0}} f(u) u d \Gamma \\
= & \frac{1}{2} \int_{\Omega} A_{t}(x, t)|u(t)|^{2} d x-2 E(t) \\
& -\left(\int_{0}^{t} g(s) d s\right) \int_{\Omega}|\sqrt{a(x)} \nabla u|^{2} d x \\
& +\int_{\Omega}(g \circ \nabla u) d x+2 \int_{\Gamma_{0}} F(u) d \Gamma \\
& +\int_{\Omega} \int_{0}^{t} a(x) g(t-s) \nabla u(s) \nabla u(t) d s d x \\
& -\int_{\Gamma_{0}} f(u) u d \Gamma .
\end{aligned}
$$

Next, estimating some items of (25), combined with the definition of $E(t)$, we get $2 E(t) \geq \int_{\Omega} k(x, t)|\nabla u|^{2} d x$; that is,

$$
\int_{\Omega}|\nabla u|^{2} d x \leq 2 E(t)+\left(\int_{0}^{t} g(s) d s\right) \int_{\Omega}|\sqrt{a(x)} \nabla u|^{2} d x .
$$

By (H3), Cauchy inequality, and Hölder inequality, we have that

$$
\begin{aligned}
\left|\int_{\Omega} \int_{0}^{t} a(x) g(t-s) \nabla u(s) \nabla u(t) d s d x\right| \\
\leq\left|\int_{\Omega} \int_{0}^{t} a(x) g(t-s)[\nabla u(s)-\nabla u(t)] d s d x\right| \\
+\left.\left|\int_{\Omega} \int_{0}^{t} g(t-s)\right| \sqrt{a(x)} \nabla u(t)\right|^{2} d s d x \mid \\
\leq \frac{1}{2 \eta} \int_{\Omega}\left(\int_{0}^{t} g^{1 / 2}(t-s) g^{1 / 2}(t-s) \sqrt{a(x)}\right. \\
\quad+\eta \int_{\Omega}|\sqrt{a(x)} \nabla u(t)|^{2} d x+\left(\int_{0}^{t} g(s) d s\right) \\
\quad \times \int_{\Omega}|\sqrt{a(x)} \nabla u|^{2} d x \\
\leq \frac{1}{2 \eta} \int_{\Omega}\left(\int_{0}^{t} g(t-s) d s \int_{0}^{t} g(t-s)\right. \\
+\eta \int_{\Omega}|\sqrt{a(x)} \nabla u(t)|^{2} d x \\
\left.\times|\sqrt{a(x)}(\nabla u(s)-\nabla u(t))|^{2} d s\right) d x
\end{aligned}
$$

$$
\begin{aligned}
& +\left(\int_{0}^{t} g(s) d s\right) \int_{\Omega}|\sqrt{a(x)} \nabla u|^{2} d x \\
& \leq \eta\|a\|_{L^{\infty}} \int_{\Omega}|\nabla u(t)|^{2} d x \\
& +\frac{\|g\|_{L^{1}}}{2 \eta} \int_{\Omega} \int_{0}^{t} g(t-s) d s \\
& \times|\sqrt{a(x)}(\nabla u(s)-\nabla u(t))|^{2} d s d x \\
& +\|a\|_{L^{\infty}}\|g\|_{L^{1}} \int_{\Omega}|\nabla u(t)|^{2} d x \\
& \leq\|a\|_{L^{\infty}}\left(\eta+\|g\|_{L^{1}}\right) \int_{\Omega}|\nabla u(t)|^{2} d x \\
& +\frac{\|g\|_{L^{1}}}{2 \eta} \int_{\Omega}(g \circ \nabla u) d x \\
& \leq 2\|a\|_{L^{\infty}}\left(\eta+\|g\|_{L^{1}}\right) E(t) \\
& +\frac{\|g\|_{L^{1}}}{2 \eta} \int_{\Omega}(g \circ \nabla u) d x \\
& +\|a\|_{L^{\infty}}\left(\eta+\|g\|_{L^{1}}\right)\left(\int_{0}^{t} g(s) d s\right) \\
& \times \int_{\Omega}|\sqrt{a(x)} \nabla u(t)|^{2} d x .
\end{aligned}
$$

Combining this with (H2), (H3), (25), (27), and Lemma 4, we get

$$
\begin{aligned}
\frac{d}{d t} \varphi(t) \leq & -\left[2-2\|a\|_{L^{\infty}}\left(\eta+\|g\|_{L^{1}}\right)\right] E(t) \\
& +\left(1+\frac{\|g\|_{L^{1}}}{2 \eta}\right) \int_{\Omega}(g \circ \nabla u) d x-\int_{\Gamma_{0}} f(u) u d \Gamma \\
& -\left[1-\|a\|_{L^{\infty}}\left(\eta+\|g\|_{L^{1}}\right)\right]\left(\int_{0}^{t} g(s) d s\right) \\
& \times \int_{\Omega}|\sqrt{a(x)} \nabla u(t)|^{2} d x+2 \int_{\Gamma_{0}} F(u) d \Gamma \\
\leq & -\left[2-2\|a\|_{L^{\infty}}\left(\eta+\|g\|_{L^{1}}\right)\right] E(t) \\
& +\left(1+\frac{\|g\|_{L^{1}}}{2 \eta}\right) \int_{\Omega}(g \circ \nabla u) d x \\
& -\left[1-\|a\|_{L^{\infty}}\left(\eta+\|g\|_{L^{1}}\right)\right]\left(\int_{0}^{t} g(s) d s\right) \\
& \times \int_{\Omega}|\sqrt{a(x)} \nabla u(t)|^{2} d x .
\end{aligned}
$$


For convenience, we take

$$
\begin{aligned}
& \theta_{1}(\eta)=2-2\|a\|_{L^{\infty}}\left(\eta+\|g\|_{L^{1}}\right), \\
& \theta_{2}(\eta)=1+\frac{\|g\|_{L^{1}}}{2 \eta}, \\
& \theta_{3}(\eta)=\left[1-\|a\|_{L^{\infty}}\left(\eta+\|g\|_{L^{1}}\right)\right]\left(\int_{0}^{t} g(s) d s\right) .
\end{aligned}
$$

Clearly, $\theta_{2}(\eta)>0$, for $\eta>0$. We have to take appropriate $\eta$ to ensure that $\theta_{1}(\eta)>0$ and $\theta_{3}(\eta)>0$. First, if $\theta_{1}(\eta)>0$, that is, $2-2\|a\|_{L^{\infty}}\left(\eta+\|g\|_{L^{1}}\right)>0$, we can get $\eta<1 /\|a\|_{L^{\infty}}-\|g\|_{L^{1}}$. Next, if $\theta_{3}(\eta)>0$, that is, $\left[1-\|a\|_{L^{\infty}}\left(\eta\|g\|_{L^{1}}\right)\right]\left(\int_{0}^{t} g(s) d s\right)>0$, noting that $\int_{0}^{t} g(s) d s \leq\|g\|_{L^{1}}$ and $\int_{0}^{t} g(s) d s \geq \int_{0}^{T} g(s) d s:=$ $g_{0}>0$ for $t \geq T>0$, we get

$$
\begin{aligned}
0 & <\left[1-\|a\|_{L^{\infty}}\left(\eta+\|g\|_{L^{1}}\right)\right]\left(\int_{0}^{t} g(s) d s\right) \\
& =\int_{0}^{t} g(s) d s-\|a\|_{L^{\infty}}\left(\eta+\|g\|_{L^{1}}\right)\left(\int_{0}^{t} g(s) d s\right) \\
& \leq\|g\|_{L^{1}}-g_{0}\|a\|_{L^{\infty}}\left(\eta+\|g\|_{L^{1}}\right) \\
& =\|g\|_{L^{1}}-g_{0}\|a\|_{L^{\infty}}\|g\|_{L^{1}}-g_{0}\|a\|_{L^{\infty}} \eta,
\end{aligned}
$$

so we can take

$$
\eta<\frac{\|g\|_{L^{1}}-g_{0}\|a\|_{L^{\infty}}\|g\|_{L^{1}}}{g_{0}\|a\|_{L^{\infty}}}
$$

For some $T>0$, we take positive constant $\eta_{0}$ such that

$$
\begin{array}{r}
\eta_{0}=\frac{1}{2} \min \left\{\frac{1}{\|a\|_{L^{\infty}}}-\|g\|_{L^{1}}, \frac{\|g\|_{L^{1}}-g_{0}\|a\|_{L^{\infty}}\|g\|_{L^{1}}}{g_{0}\|a\|_{L^{\infty}}}\right\}, \\
\forall t \geq T ;
\end{array}
$$

then we have $k_{1}=\theta_{1}\left(\eta_{0}\right)>0, k_{2}=\theta_{2}\left(\eta_{0}\right)>0$ for $t \geq T$, and

$$
\frac{d}{d t} \varphi(t) \leq-k_{1} E(t)+k_{2} \int_{\Omega}(g \circ \nabla u) d x .
$$

This completes the proof.

\section{Global Existence and Uniqueness}

In this section, we show the existence and uniqueness of the global solution to problem (1)-(2) by the Galerkin method, contraction mapping principle, and contradiction argument.

Theorem 6. Assume that (H1)-(H4) holds; there exists a unique global solution of the problem (1)-(2).
Proof

Step 1. We consider the following auxiliary problem for a given $v$ :

$$
\begin{gathered}
A(x, t) u_{t}-\Delta u+\int_{0}^{t} g(t-s) \operatorname{div}[a(x) \nabla u(s)] d s=0, \\
(x, t) \in \Omega \times(0, \infty), \\
-\frac{\partial u}{\partial \nu}+\int_{0}^{t} g(t-s)[a(x) \nabla u(s) \cdot v] d s=f(v), \\
(x, t) \in \Gamma_{0} \times[0, \infty), \\
u(x, t)=0, \quad(x, t) \in \Gamma_{1} \times(0, \infty), \\
u(x, 0)=u_{0}(x), \quad x \in \Omega,
\end{gathered}
$$

where $u$ is the solution that we required. Giving some $T>0$, we will consider the solution of the problem (34) in the space $S=C([0, T] ; V) \cap C^{1}\left([0, T] ; L^{1}(\Omega)\right)$ and define the norm as $\|u\|_{S}^{2}=\max _{t \in[0, T]}\|\nabla u\|_{2}^{2}$.

Step 2. We will show that with the hypotheses (H1)-(H4), for $T>0, v \in S$, there exists a unique $u \in S$ which satisfies (34).

Choose the basis $\left\{w_{j}\right\}_{j \geq 1}$ in $V \cap H^{2}(\Omega)$, which are orthonormal in $L^{2}(\Omega)$ and let $V_{m}=\operatorname{span}\left\{w_{1}, \ldots, w_{m}\right\}$ be the subspace of $V \cap H^{2}(\Omega)$ generated by the first $m$ vectors. For any $m \in N$, define

$$
u_{m}(t)=\sum_{j=1}^{m} \zeta_{j m} w_{j}
$$

where $u_{m}(t)$ satisfies the following equation:

$$
\begin{aligned}
& \left(A(x, t) u_{m}^{\prime}, w\right)+\left(\nabla u_{m}, \nabla w\right) \\
& \quad-\int_{0}^{t} a(x) g(t-s)\left(\nabla u_{m}(s), \nabla w\right) d s \\
& \quad+(f(v), w)_{\Gamma_{0}}=0,
\end{aligned}
$$

with the initial condition

$$
u_{m}(0)=u_{0 m}=\sum_{j=1}^{m}\left(u_{m}(0), w_{j}\right) w_{j},
$$

for any $w \in V$. By standard nonlinear ODE theory, we know that the problem (36) has a unique solution on some interval $\left[0, T_{m}\right]$. The extension of the solution to the whole interval $[0, T)$ is a consequence of the first estimate, which we are going to prove below. Taking $w=u_{m}^{\prime}(t)$, we get

$$
\begin{aligned}
& \left(A(x, t) u_{m}^{\prime}, u_{m}^{\prime}\right)+\left(\nabla u_{m}, \nabla u_{m}^{\prime}\right) \\
& \quad-\int_{0}^{t} a(x) g(t-s)\left(\nabla u_{m}(s), \nabla u_{m}^{\prime}(t)\right) d s \\
& \quad+\left(f(v), u_{m}^{\prime}(t)\right)_{\Gamma_{0}}=0 ;
\end{aligned}
$$


that is,

$$
\begin{aligned}
& \int_{\Omega} A(x, t)\left|u_{m}^{\prime}(t)\right|^{2} d x+\frac{d}{d t}\left[\frac{1}{2}\left\|\nabla u_{m}(t)\right\|_{2}^{2}\right] \\
& +\frac{1}{2} \int_{\Omega}\left[a(x) g(t)\left|\nabla u_{m}(t)\right|^{2}-\left(g^{\prime} \circ \nabla u_{m}(t)\right)\right] d x \\
& +\frac{1}{2} \frac{d}{d t} \int_{\Omega}\left[\left(g \circ \nabla u_{m}(t)\right)\right. \\
& \left.\quad-\int_{0}^{t} g(s)\left|\sqrt{a(x)} \nabla u_{m}(t)\right|^{2} d s\right] d x \\
& +\left(f(v), u_{m}^{\prime}(t)\right)_{\Gamma_{0}}=0,
\end{aligned}
$$

then, we have

$$
\begin{aligned}
\int_{\Omega} A(x, t)\left|u_{m}^{\prime}(t)\right|^{2} d x \\
\quad+\frac{d}{d t}\left[\frac{1}{2}\left(1-a(x) \int_{0}^{t} g(s) d s\right)\left\|\nabla u_{m}(t)\right\|_{2}^{2}\right] \\
\quad+\frac{1}{2} \frac{d}{d t} \int_{\Omega}\left(g \circ \nabla u_{m}(t)\right)(t) d x \\
\quad+\frac{1}{2} g(t)\left\|\sqrt{a(x)} \nabla u_{m}(t)\right\|_{2}^{2} \\
\quad-\frac{1}{2} \int_{\Omega}\left(g^{\prime} \circ \nabla u_{m}(t)\right)(t) d x+\left(f(v), u_{m}^{\prime}(t)\right)_{\Gamma_{0}}=0 .
\end{aligned}
$$

Integrating (40) over $(0, t), t \in\left[0, T_{m}\right]$, we get

$$
\begin{aligned}
\int_{0}^{t} \int_{\Omega} & A(x, t)\left|u_{m}^{\prime}(t)\right|^{2} d x d t \\
& +\frac{1}{2}\left(1-a(x) \int_{0}^{t} g(s) d s\right)\left\|\nabla u_{m}(t)\right\|_{2}^{2} \\
& +\frac{1}{2} \int_{\Omega}\left(g \circ \nabla u_{m}\right)(t) d x-\frac{1}{2}\left\|\nabla u_{m}(0)\right\|_{2}^{2} \\
& +\int_{0}^{t} g(s)\left\|\sqrt{a(x)} \nabla u_{m}(s)\right\|_{2}^{2} d s \\
& -\frac{1}{2} \int_{0}^{t} \int_{\Omega}\left(g^{\prime} \circ \nabla u_{m}\right)(t) d x d t \\
& +\int_{0}^{t} \int_{\Gamma_{0}} f(v) u_{m}^{\prime}(t) d \Gamma d t=0 .
\end{aligned}
$$

Next, estimating some items of (41), by (H1), we obtain

$$
\begin{gathered}
\int_{\Omega}\left(g \circ \nabla u_{m}\right)(t) d x-\int_{0}^{t} \int_{\Omega}\left(g^{\prime} \circ \nabla u_{m}\right)(t) d x d t \\
+\int_{0}^{t} g(s)\left\|\sqrt{a(x)} \nabla u_{m}(s)\right\|_{2}^{2} d s \geq 0 .
\end{gathered}
$$

By the Hölder inequality, $p /(p+1)+1 /(p+1)=1$ and $H_{0}^{1}(\Omega) \hookrightarrow L^{p+1}(\Omega), H_{0}^{1}\left(\Gamma_{0}\right) \hookrightarrow L^{2 p}\left(\Gamma_{0}\right)$, we have

$$
\begin{gathered}
\int_{0}^{t} \int_{\Gamma_{0}} f(v) u_{m}^{\prime}(t) d \Gamma d t \leq \int_{0}^{t}\|f(v)\|_{p+1, \Gamma_{0}}^{p}\left\|u_{m}^{\prime}(t)\right\|_{p+1, \Gamma_{0}} d t \\
\leq \frac{1}{4 \eta} \int_{0}^{t}\|f(v)\|_{p+1, \Gamma_{0}}^{p} d t+\eta \int_{0}^{t}\left\|\nabla u_{m}^{\prime}(t)\right\|_{2}^{2} d t \\
\leq \eta \int_{0}^{t}\left\|\nabla u_{m}^{\prime}(t)\right\|_{2}^{2} d t+C(T) .
\end{gathered}
$$

By assumption of the boundedness of $A(x, t)$ and Sobolev embedding inequality, we get

$$
\int_{0}^{t} \int_{\Omega} A(x, t)\left|u_{m}^{\prime}(t)\right|^{2} d x d t \leq C(T)+C_{\delta} \int_{0}^{t}\left\|u_{m}^{\prime}(t)\right\|_{2}^{2} d t .
$$

Substituting the estimates (42)-(44) into (41), we obtain

$$
\begin{gathered}
C_{\delta} \int_{0}^{t}\left\|u_{m}^{\prime}(t)\right\|_{2}^{2} d t+\frac{1}{2} k(x, t)\left\|\nabla u_{m}(t)\right\|_{2}^{2} \\
+\eta \int_{0}^{t}\left\|u_{m}^{\prime}(t)\right\|_{2}^{2} d t \leq C(T) .
\end{gathered}
$$

Hence, there exists a subsequence of $\left\{u_{m}\right\}$, which will be still denoted by $\left\{u_{m}\right\}$, such that

$$
\begin{aligned}
& u_{m}^{\prime} \rightarrow u \text { weak-star in } L^{\infty}([0, T] ; V), \\
& u_{m}^{\prime} \rightarrow u^{\prime} \text { weak-star in } L^{\infty}\left([0, T] ; L^{1}(\Omega)\right), \\
& u_{m}^{\prime} \rightarrow u^{\prime} \text { weak-star in } L^{2}\left([0, T) \times H_{0}^{1}(\Omega)\right) \cap L^{m+2}([0, T] \times \\
& \left.\Gamma_{0}\right) .
\end{aligned}
$$

Noting that $u \in H^{1}([0, T] ; V)$, we can get $u \in C([0, T] ; V)$. The existence of solution $u$ is proved.

Next, we will prove the uniqueness of the solution $u$ of (34) by contradiction argument. Let $u_{1}, u_{2}$ be two solutions of problem (34) with the same initial values. Letting that $U=$ $u-u^{-}$and taking $U$ into (41), we have

$$
\begin{aligned}
\int_{0}^{t} \int_{\Omega} & A(x, t)\left|U_{t}(t)\right|^{2} d x d t \\
+ & \frac{1}{2}\left(1-a(x) \int_{0}^{t} g(s) d s\right)\|\nabla U(t)\|_{2}^{2} \\
& +\frac{1}{2} \int_{\Omega}(g \circ \nabla U)(t) d x \\
+ & \frac{1}{2} \int_{0}^{t} g(s)\|\sqrt{a(x)} \nabla U(s)\|_{2}^{2} d s \\
+ & \left\{-\frac{1}{2} \int_{\Omega} \int_{0}^{t}\left(g^{\prime} \circ \nabla U\right)(s) d s d x\right\}=0 .
\end{aligned}
$$

By $(\mathrm{H} 1)-(\mathrm{H} 3)$, each term of the left-hand side is nonnegative; then $u=u^{-}$follows immediately.

Step 3 (local existence and uniqueness). In this step, we will derive existence and uniqueness of local solution to problem 
(1)-(2) for appropriate small time $T$ by using contraction mapping theorem. That is,

$$
\begin{aligned}
& \left(A(x, t) u_{t}, \omega\right)+(\nabla u, \nabla \omega) \\
& \quad-\int_{0}^{t} a(x) g(t-s)(\nabla u(s), \nabla \omega) d s \\
& \quad+(f(u), \omega)_{\Gamma_{0}}=0,
\end{aligned}
$$

such that

$$
u \in C([0, T] ; V) \cap C^{1}\left([0, T] ; L^{1}(\Omega)\right) .
$$

For $R>0, T>0$, we define

$$
B_{R}=\left\{u \in S: u(0)=0,\|u\|_{s} \leq R\right\} .
$$

$B_{R}$ is nonempty for taking $R$ sufficiently large. We define a mapping $F: u=F(v)$ from $B_{R}$ to $S$.

Firstly, we will prove that $F$ is a contraction mapping from $B_{R}$ to itself. From Lemma 2, we know that for any fixed $v \in$ $B_{R}$, the solution satisfies the following equation:

$$
\begin{aligned}
\int_{0}^{t} \int_{\Omega} & A(x, t)\left|u_{t}\right|^{2} d x d t+\frac{1}{2}\left(1-a(x) \int_{0}^{t} g(s) d s\right)\|\nabla u\|_{2}^{2} \\
& +\frac{1}{2} \int_{\Omega}(g \circ \nabla u)(t) d x-\frac{1}{2}\|\nabla u(0)\|_{2}^{2} \\
& +\frac{1}{2} \int_{0}^{t} g(s)\|\sqrt{a(x)} \nabla u(s)\|_{2}^{2} d s \\
& -\frac{1}{2} \int_{\Omega} \int_{0}^{t}\left(g^{\prime} \circ \nabla u\right)(s) d s d x \\
+ & \int_{0}^{t} \int_{\Gamma_{0}} f(v) u_{t}(t) d \Gamma d t=0 .
\end{aligned}
$$

Similar to the estimates of (42) and (43), we obtain

$$
\begin{gathered}
C_{\delta} \int_{0}^{t}\left\|u_{t}(t)\right\|_{2}^{2} d t+\frac{1}{2} k(x, t)\|\nabla u\|_{2}^{2} \\
+\eta \int_{0}^{t}\left\|\nabla u^{\prime}(t)\right\|_{2}^{2} d t \\
\leq \frac{1}{4 \eta} T\|\nabla v\|_{2}^{2 p} \leq \frac{1}{4 \eta} T R^{2 p}
\end{gathered}
$$

selecting $T$ sufficiently small, then we have

$$
\|u(s)\|_{S}^{2} \leq R^{2}
$$

for taking $T$ sufficiently small, so $F$ is a mapping from $B_{R}$ to itself.
Secondly, we will prove that $F$ is a contraction mapping. Let $U=u-u^{-}, V=v-v^{-}$, where $F(v)=u, F\left(v^{-}\right)=u^{-}$; then for any $w \in V$, we have

$$
\begin{aligned}
& \left(A(x, t) U_{t}, w\right)+(\nabla U, \nabla w) \\
& \quad-\int_{0}^{t} a(x) g(t-s)(\nabla U(s), \nabla w) d s \\
& +\int_{\Gamma_{0}}\left(f(v)-f\left(v^{-}\right)\right) w d \Gamma=0 .
\end{aligned}
$$

Taking $w=U_{t}$ and integrating over $(0, t]$, we get

$$
\begin{aligned}
\int_{0}^{t} \int_{\Omega} & A(x, t)\left|U_{t}\right|^{2} d x d t+\frac{1}{2}\left(1-a(x) \int_{0}^{t} g(s) d s\right)\|\nabla U\|_{2}^{2} \\
& +\frac{1}{2} \int_{\Omega}(g \circ \nabla U)(t) d x \\
& +\frac{1}{2} \int_{0}^{t} g(s)\|\sqrt{a(x)} \nabla U(s)\|_{2}^{2} d s \\
& -\frac{1}{2} \int_{\Omega} \int_{0}^{t}\left(g^{\prime} \circ \nabla u\right)(s) d s d x \\
& +\int_{0}^{t} \int_{\Gamma_{0}}\left(f(v)-f\left(v^{-}\right) U_{t}(t) d \Gamma d t=0 .\right.
\end{aligned}
$$

By (H3), we obtain

$$
\begin{aligned}
\int_{\Gamma_{0}}\left(f(v)-f\left(v^{-}\right) U_{t}(t) d \Gamma\right. & \leq C_{1} \int_{\Gamma_{0}}\left|v-v^{-}\right|\left|U_{t}(t)\right| d \Gamma \\
& \leq C_{2}\left\|U_{t}(t)\right\|_{2}\left\|\nabla V^{-}(t)\right\|_{2, \Gamma_{0}} \\
& \leq C_{3}\left\|U_{t}(t)\right\|_{2}\left\|\nabla V^{-}(t)\right\|_{2, \Gamma_{0}}
\end{aligned}
$$

where $\xi$ is located between $v$ and $v^{-}$. Combining of (42), (55), and (54) yields

$$
\begin{gathered}
\int_{0}^{t} \int_{\Omega} A(x, t)\left|U_{t}(t)\right|^{2} d x d t+\frac{1}{2}\left(1-a(x) \int_{0}^{t} g(s) d s\right) \\
\times\|\nabla U\|_{2}^{2} \leq C_{3} \int_{0}^{t}\left\|U_{t}(t)\right\|_{2}\left\|\nabla V^{-}(t)\right\|_{2, \Gamma_{0}} ;
\end{gathered}
$$

that is

$$
\|U\|_{S} \leq C_{4} T R^{p}\left\|V^{-}\right\|_{S}
$$

Taking $T$ sufficiently small such that $C_{4} T R^{p}<1, F$ is a contraction mapping.

Step 4. We show that if $T_{\max }=\sup \{T>0: u=u(t)$ exists on $[0, T]\}<\infty$, then $\lim _{t \rightarrow T_{\max }^{-}}\|u\|_{S}^{2}=\infty$.

We will use a standard continuation argument to prove it. Indeed, by contradiction argument, suppose that $T_{\max }<\infty$ and $\lim _{t \rightarrow T_{\max }^{-}}\|u\|_{S}^{2}<\infty$; then there exists a sequence $\left\{t_{n}, n=\right.$ 
$1,2, \ldots\}$ and a constant $K>0$, such that $t_{n} \rightarrow T_{\max }$ as $n \rightarrow$ $+\infty$ and $\left\|u\left(t_{n}\right)\right\|_{S}^{2} \leq K, n=1,2, \ldots$. As we have already shown previously, for each $n \in N$ there exists a unique solution of the problem (1)-(2) with initial data $u\left(t_{n}\right)$ on $\left[t_{n}, t_{n}+T^{*}\right]$, where $T^{*}>0$ depends on $K$ and is independent of $n \in N$. Thus, for $n \in N$ large enough, we can get $T_{\max }<t_{n}+T^{*}$. This contradicts the maximality of $T_{\max }$.

Step 5. In the final step, we only need to prove the existence of the global solution. By (H3) and Poincaré inequality, we have

$$
\begin{aligned}
E(0) \geq E(t)= & \frac{1}{2} k(x, t) \int_{\Omega}|\nabla u|^{2} d x \\
& +\frac{1}{2} \int_{\Omega}(g \circ \nabla u)(t) d x+\int_{\Gamma_{0}} F(u) d \Gamma \\
\geq & \frac{1}{2}\left(1-a(x) \int_{0}^{t} g(s) d s\right)\|\nabla u\|_{2}^{2} \\
& +\frac{1}{2} \int_{\Omega}(g \circ \nabla u)(t) d x \\
\geq & C\|u\|_{S}^{2}, \quad \forall t \geq 0 .
\end{aligned}
$$

It is easy to see that $T_{\max }=\infty$. This completes the proof.

\section{Uniform Energy Decay Rates}

In this section, we establish the estimate of uniform energy decay rates and make use of the above assumptions and preliminaries to prove the results.

Theorem 7. Assume that (H1)-(H4) hold and there exists a positive constant $c>0$ such that $g^{\prime}(t) \leq-c g(t)$. If $u_{0} \in H^{1}(\Omega)$, then for some $T>0$, there exists a positive constant $C_{0}$ such that the solution of (1)-(2) satisfies

$$
E(t) \leq 4 E(0) e^{-c_{0} t}, \quad \forall t \geq T
$$

Proof. Let $\gamma>0$ be a positive constant. We introduce

$$
\psi(t)=\gamma E(t)+\varphi(t)
$$

since $|\varphi(t)| \leq C E(t)$, we get

$$
\frac{\gamma}{2} E(t) \leq \psi(t) \leq 2 \gamma E(t), \quad \forall t \geq 0
$$

by taking $\gamma$ large enough. From (18) and the assumption of $g^{\prime}(t) \leq-c g(t)$, applying Lemma 3 and taking $\gamma$ large enough and $t \geq T$, we obtain that

$$
\begin{aligned}
\frac{d}{d t} \psi(t)= & \gamma \frac{d}{d t} E(t)+\frac{d}{d t} \varphi(t) \\
\leq & \gamma\left(\frac{1}{2} \int_{\Omega}\left(g^{\prime} \circ \nabla u-g(t)|\sqrt{a(x)} \nabla u|^{2}\right) d x\right. \\
& -\int_{\Omega} A(x, t)\left|u_{t}\right|^{2} d x-k_{1} E(t)+k_{2} \int_{\Omega}(g \circ \nabla u) d x \\
\leq & -\left(\frac{c \gamma}{2}-k_{2}\right) \int_{\Omega}(g \circ \nabla u) d x \\
& -\frac{\gamma}{2} \int_{\Omega} g(t)|\sqrt{a(x)} \nabla u|^{2} d x \\
& -\gamma \int_{\Omega} A(t)\left|u_{t}\right|^{2} d x-k_{1} E(t) \\
\leq & -k_{1} E(t) \leq-\frac{k_{1}}{2 \gamma} \psi(t)
\end{aligned}
$$

by the Gronwall's inequality which implies that

$$
\psi(t) \leq \psi(0) e^{-\left(k_{1} / 2 \gamma\right) t}, \quad \forall t \geq T .
$$

Using (61), we obtain

$$
E(t) \leq 4 E(0) e^{-\left(k_{1} / 2 \gamma\right) t}, \quad \forall t \geq T .
$$

This completes the proof.

Theorem 8. Assume that (H1)-(H4) hold, and there exists a positive constant $c>0$ such that $g^{\prime}(t) \leq-c g^{1+1 / q}(t), q>2$. If $u_{0} \in H^{1}(\Omega)$, then for some $T>0$ there exists a positive constant $C_{\omega}$, such that the solution of (1)-(2) satisfies

$$
E(t) \leq \frac{C_{\omega}}{(1+t)^{q}}, \quad \forall t \geq T .
$$
lemma.

In order to prove Theorem 8 , we first quote the following

Lemma 9. Assume that $v \in L^{\infty}\left(0, T ; H^{1}(\Omega)\right)$ and $g$ is a continuous function. Then there exists a positive constant $C$, such that

$$
\begin{aligned}
\int_{\Omega}(g \circ \nabla v) d x \leq & C\left(t\|v(t)\|_{H^{1}}^{2}+\int_{0}^{t}\|v(t)\|_{H^{1}}^{2} d s\right)^{1 /(q+1)} \\
& \times\left(\int_{\Omega} g^{1+1 / q} \circ \nabla v d x\right)^{q /(q+1)} .
\end{aligned}
$$

Moreover,

$$
\int_{0}^{\infty} g^{1-\theta}(s) d s<\infty, \quad 0<\theta<1 .
$$


Then we have

$$
\begin{aligned}
\int_{\Omega}(g \circ \nabla v) d x \leq & C\left(\int_{0}^{\infty} g^{1-\theta}(s) d s\|v\|_{L^{\infty}\left(0, T ; H^{1}(\Omega)\right)}^{2}\right)^{1 /(\theta q+1)} \\
& \times\left(\int_{\Omega} g^{1+1 / q} \circ \nabla v d x\right)^{\theta q /(\theta q+1)}
\end{aligned}
$$

Proof. Applying the Hölder inequality, we obtain

$$
\begin{aligned}
\int_{\Omega}(g \circ \nabla v) d x= & \int_{\Omega} \int_{0}^{t} g(t-s) \\
& \times|\sqrt{a(x)}(\nabla v(t)-\nabla v(s))|^{2} d s d x \\
\leq & \left(\int_{\Omega} \int_{0}^{t}|g(s)|^{1-\theta}|\omega(s)| d s d x\right)^{1 /(\theta q+1)} \\
& \times\left(\int_{\Omega} \int_{0}^{t}|g(s)|^{1+1 / q}|\omega(s)| d s d x\right)^{\theta q /(\theta q+1)} \\
\leq & \left(\int_{\Omega} g^{1-\theta} \circ \nabla v d x\right)^{1 /(\theta q+1)} \\
& \times\left(\int_{\Omega} g^{1+1 / q} \circ \nabla v d x\right)^{\theta q /(\theta q+1)}
\end{aligned}
$$

where $\omega(s)=|\sqrt{a(x)}(\nabla v(t)-\nabla v(s))|^{2}$. Noting that $\int_{0}^{\infty} g^{1-\theta}(s) d s<\infty, 0<\theta<1$, we obtain

$$
\begin{aligned}
\int_{\Omega} g^{1-\theta} \circ \nabla v d x= & \int_{0}^{t} g^{1-\theta}(t-s) \\
& \times \int_{\Omega}|\sqrt{a(x)}(\nabla v(t)-\nabla v(s))|^{2} d x d s \\
\leq & C \int_{0}^{t} g^{1-\theta}(s) d s\|v\|_{L^{\infty}\left(0, T ; H^{1}(\Omega)\right)}^{2}
\end{aligned}
$$

which implies that

$$
\begin{aligned}
\int_{\Omega} g \circ \nabla v d x \leq & \left.C\left(\int_{0}^{t} g^{1-\theta}(s) d s\right)\|v\|_{L^{\infty}\left(0, T ; H^{1}(\Omega)\right)}^{2}\right)^{1 /(\theta q+1)} \\
& \times\left(\int_{\Omega} g^{1+1 / q} \circ \nabla v d x\right)^{\theta q /(\theta q+1)}
\end{aligned}
$$

If $\theta=1$, we have

$$
\begin{aligned}
\int_{\Omega} g^{1-\theta} \circ \nabla v d x & =\int_{\Omega} 1 \circ \nabla v d x \\
& =\int_{\Omega} \int_{0}^{t}|\sqrt{a(x)}(\nabla v(t)-\nabla v(s))|^{2} d s d x \\
& \leq C\left(t \int_{\Omega}|\nabla v(t)|^{2} d x\right. \\
& \left.\quad+\int_{\Omega} \int_{0}^{t}|\nabla v(s)|^{2} d x d s\right) .
\end{aligned}
$$

Applying the above inequality and (69), we obtain

$$
\begin{aligned}
\int_{\Omega} g \circ \nabla v d x \leq & C\left(t \int_{\Omega}|\nabla v(t)|^{2} d x\right. \\
& \left.+\int_{\Omega} \int_{0}^{t}|\nabla v(s)|^{2} d x d s\right)^{1 /(q+1)} \\
& \times\left(\int_{\Omega} g^{1+(1 / q)} \circ \nabla v d x\right)^{q /(q+1)} \\
\leq & C\left(t\|v(t)\|_{H^{1}}^{2}+\int_{0}^{t}\|v(t)\|_{H^{1}}^{2} d s\right)^{1 /(q+1)} \\
& \times\left(\int_{\Omega} g^{1+(1 / q)} \circ \nabla v d x\right)^{q /(q+1)} .
\end{aligned}
$$

This completes the proof.

Proof of Theorem 8. From the assumption $g^{\prime}(t) \leq-c g^{1+1 / q}(t)$, we have $\left[g^{-1 / q}(t)\right]^{\prime} \geq c / q$. Integrating it over $[0, t]$, we get

$$
g(t) \leq C_{1}(1+t)^{-q}, \quad C_{1}>0 .
$$

Taking $\theta=1 / 2$ in Lemma 9, $(1-\theta) q=q / 2>1$, then we obtain

$$
\int_{0}^{\infty} g^{1-\theta}(s) d s \leq \int_{0}^{\infty} \frac{1}{(1+s)^{q / 2}}<\infty
$$

Substituting this estimate into (68), using (18) and Lemma 4, we have

$$
\int_{\Omega} g \circ \nabla u d x \leq C_{2} E(0)^{2 / q+2}\left(\int_{\Omega} g^{1+1 / q} \circ \nabla u d x\right)^{q /(q+2)} .
$$

Applying (76), (23), and Lemma 3 and taking $t \geq T$, we get

$$
\begin{aligned}
\frac{d}{d t} \varphi(t) \leq & -k_{1} E(t)+k_{2} \int_{\Omega}(g \circ \nabla u) d x \\
\leq & -k_{1} E(t)+k_{2} C_{2} E(0)^{2 /(q+2)} \\
& \times\left(\int_{\Omega} g^{1+1 / q} \circ \nabla u d x\right)^{q /(q+2)} .
\end{aligned}
$$


Since $|\varphi(t)| \leq C E(t)$, applying the Young's inequality, from (19) and (77), we deduce that

$$
\begin{aligned}
\frac{d}{d t}\left[E^{2 / q} \varphi\right](t)= & \frac{2}{q} \varphi(t) E^{2 / q-1}(t) \frac{d}{d t} E(t)+E^{2 / q}(t) \frac{d}{d t} \varphi(t) \\
\leq & -\frac{2}{q} C E^{2 / q}(t) \frac{d}{d t} E(t)+E^{2 / q}(t) \frac{d}{d t} \varphi(t) \\
\leq & -C_{0} \frac{d}{d t} E^{1+2 / q}(t)-k_{1} E^{1+2 / q}(t) \\
& +k_{2} C_{2} E(0)^{2 /(q+2)} E^{2 / q}(t) \\
& \times\left(\int_{\Omega} g^{1+1 / q} \circ \nabla u d x\right)^{q /(q+2)} \\
\leq & -C_{0} \frac{d}{d t} E^{1+2 / q}(t)-k_{1} E^{1+2 / q}(t) \\
& +k_{2} C_{2} E(0)^{2 / q+2} \varepsilon E^{1+2 / q}(t) \\
& +k_{2} C_{2} E(0)^{2 / q+2} C_{\varepsilon}\left(\int_{\Omega} g^{1+1 / q} \circ \nabla u d x\right) .
\end{aligned}
$$

Since $g^{\prime}(t) \leq-c g^{1+1 / q}(t)$, that is, $g^{1+(1 / q)}(t) \leq-(1 / c) g^{\prime}(t)$, then we obtain

$$
\int_{\Omega} g^{1+(1 / q)} \circ \nabla u d x \leq-\frac{1}{c} \int_{\Omega} g^{\prime} \circ \nabla u d x .
$$

It follows from Lemma 3 that

$$
\begin{aligned}
\int_{\Omega} g^{\prime} \circ \nabla u d x= & 2 \frac{d}{d t} E(t)+\frac{1}{2} \int_{\Omega} g(t)|\sqrt{a(x)} \nabla u(t)|^{2} d x \\
& +\int_{\Omega} A(x, t)\left|u_{t}\right|^{2} d x \geq 2 \frac{d}{d t} E(t)
\end{aligned}
$$

hence, we get

$$
\int_{\Omega} g^{1+(1 / q)} \circ \nabla u d x \leq-C_{3} \frac{d}{d t} E(t) .
$$

Taking $\varepsilon$ sufficiently small, using (78) and (81), we have

$$
\begin{aligned}
\frac{d}{d t}\left[E^{2 / q} \varphi\right](t) \leq & -C_{0} \frac{d}{d t} E^{1+2 / q}(t) \\
& -\frac{k_{1}}{2} E^{1+2 / q}(t)-C_{4} \frac{d}{d t} E(t) ;
\end{aligned}
$$

then we obtain

$$
\begin{array}{r}
\frac{d}{d t}\left[E^{2 / q} \varphi+C_{0} E\right](t) \leq-\frac{k_{1}}{2} E^{1+(2 / q)}(t)-C_{4} \frac{d}{d t} E(t), \\
\forall t \geq T .
\end{array}
$$

Let $\gamma>0$ be a positive constant and define that

$$
\psi(t)=\gamma E(t)+E^{2 / q}(t)\left[\varphi(t)+C_{0} E(t)\right] .
$$

Since $|\varphi(t)| \leq C E(t),(d / d t) E(t) \leq 0$, we get

$$
\frac{\gamma}{2} E(t) \leq \psi(t) \leq 2 \gamma E(t), \quad \forall t \geq 0,
$$

by taking $\gamma$ sufficiently large. Using (83) and Lemma 3 and taking $\gamma$ sufficiently large, we obtain

$$
\begin{aligned}
\frac{d}{d t} \psi(t) & =\gamma \frac{d}{d t} E(t)+\frac{d}{d t}\left\{E^{2 / q}(t)\left[\varphi(t)+C_{0} E(t)\right]\right\} \\
& \leq \gamma \frac{d}{d t} E(t)-C_{4} \frac{d}{d t} E(t)-\frac{k_{1}}{2} E^{1+2 / q}(t) \\
& =\left(\gamma-C_{4}\right) \frac{d}{d t} E(t)-\frac{k_{1}}{2} E^{1+2 / q}(t) \\
& \leq-\frac{k_{1}}{2} E^{1+(2 / q)}(t), \quad \forall t \geq T .
\end{aligned}
$$

From (85) and (86), we have

$$
\frac{d}{d t} \psi(t) \leq-C_{5} \psi^{1+2 / q}(t), \quad \forall t \geq T .
$$

Applying the Gronwall's inequality, we get

$$
\psi(t) \leq \frac{C_{6}}{(1+t)^{q / 2}}, \quad \forall t \geq T
$$

hence

$$
E(t) \leq \frac{C_{7}}{(1+t)^{q / 2}}, \quad \forall t \geq T .
$$

Since $q>2$, we have

$$
\begin{gathered}
\int_{0}^{\infty} E(s) d s \leq \int_{0}^{\infty} \frac{C_{7}}{(1+t)^{q / 2}} d s<\infty, \\
t E(t) \leq \frac{C_{7} t}{(1+t)^{q / 2}}<\infty,
\end{gathered}
$$

and then we obtain

$$
\begin{aligned}
\int_{0}^{\infty}\|u(s)\|_{H^{1}}^{2} d s+t\|u(t)\|_{H^{1}}^{2} \\
\quad \leq C_{8}\left(\int_{0}^{\infty} E(s) d s+t E(t)\right)<\infty .
\end{aligned}
$$

Using Lemma 9, we get

$$
\int_{\Omega} g \circ \nabla u d x \leq C_{9}\left(\int_{\Omega} g^{1+1 / q} \circ \nabla u d x\right)^{q /(q+1)} .
$$

By (92) and replacing the left-hand side term of (78) by $(d / d t)\left[E^{1 / q} \varphi\right](t)$, we deduce that

$$
E(t) \leq \frac{C_{w}}{(1+t)^{q}}, \quad \forall t \geq T .
$$

This completes the proof. 


\section{Conflict of Interests}

The authors declare that they have no conflict of interests.

\section{Authors' Contribution}

All authors contributed equally to the paper and read and approved the final paper.

\section{Acknowledgments}

This work is supported by the Natural Science Foundation of Shandong Province of China (ZR2012AM018) and the Fundamental Research Funds for the Central Universities (no. 201362032). The authors would like to deeply thank all the reviewers for their insightful and constructive comments.

\section{References}

[1] Z. Tan, "The reaction-diffusion equation with Lewis function and critical Sobolev exponent," Journal of Mathematical Analysis and Applications, vol. 272, no. 2, pp. 480-495, 2002.

[2] G. Da Prato and M. Iannelli, "Existence and regularity for a class of integrodifferential equations of parabolic type," Journal of Mathematical Analysis and Applications, vol. 112, no. 1, pp. 3655, 1985.

[3] A. Friedman, The IMA Volumes in Mathematics and Its Applications, vol. 49 of Mathematics in Industrial Problems, Springer, New York, NY, USA, 1992.

[4] J. A. Nohel, "Nonlinear Volterra equations for heat flow in materials with memory," in Integral and Functional Differential Equations, T. L. Herdman, H. W. Stech, and S. M. Rankin, Eds., vol. 67 of Lecture Notes in Pure and Applied Mathematics, pp. 3-82, Marcel Dekker, New York, NY, USA, 1981.

[5] H. M. Yin, "On parabolic Volterra equations in several space dimensions," SIAM Journal on Mathematical Analysis, vol. 22, no. 6, pp. 1723-1737, 1991.

[6] H. M. Yin, "Weak and classical solutions of some nonlinear Volterra integro-differential equations," Communications in Partial Differential Equations, vol. 17, pp. 1369-1385, 1992.

[7] H. A. Levine, "Some nonexistence and instability theorems for solutions of formally parabolic equations of the form $P u_{t}=$ $-A u+F(u), "$ Archive for Rational Mechanics and Analysis, vol. 51, no. 5, pp. 371-386, 1973.

[8] V. K. Kalantarov and O. A. Ladyzhenskaya, "The occurrence of collapse for quasilinear equations of parabolic and hyperbolic types," Journal of Soviet Mathematics, vol. 10, no. 1, pp. 53-70, 1978.

[9] H. A. Levine, S. R. Park, and J. Serrin, "Global existence and nonexistence theorems for quasilinear evolution equations of formally parabolic type," Journal of Differential Equations, vol. 142, no. 1, pp. 212-229, 1998.

[10] P. Pucci and J. Serrin, "Asymptotic stability for nonlinear parabolic systems," in Energy Methods in Continuum Mechanics, pp. 66-74, Kluwer Academic Publishers, Dodrecht, The Netherlands, 1996.

[11] S. Berrimi and S. A. Messaoudi, "A decay result for a quasilinear parabolic system," Progress in Nonlinear Differential Equations and Their Applications, vol. 53, pp. 43-50, 2005.

[12] S. A. Messaoudi, "Blow-up of solutions of a semilinear heat equation with a Visco-elastic term," Progress in Nonlinear
Differential Equations and Their Applications, vol. 64, pp. 351356, 2005.

[13] Z. B. Fang and L. Sun, "Blow up of solutions with positive initial energy for the nonlocal semilinear heat equation," The KSIAM Journal, vol. 16, no. 4, pp. 235-242, 2012.

[14] S. A. Messaoudi and B. Tellab, "A general decay result in a quasilinear parabolic system with viscoelastic term," Applied Mathematics Letters, vol. 25, no. 3, pp. 443-447, 2012. 


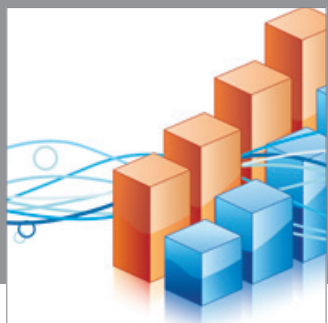

Advances in

Operations Research

mansans

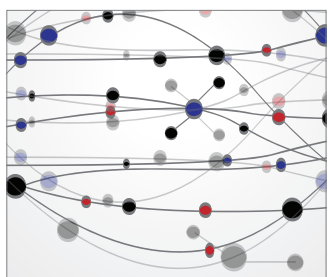

The Scientific World Journal
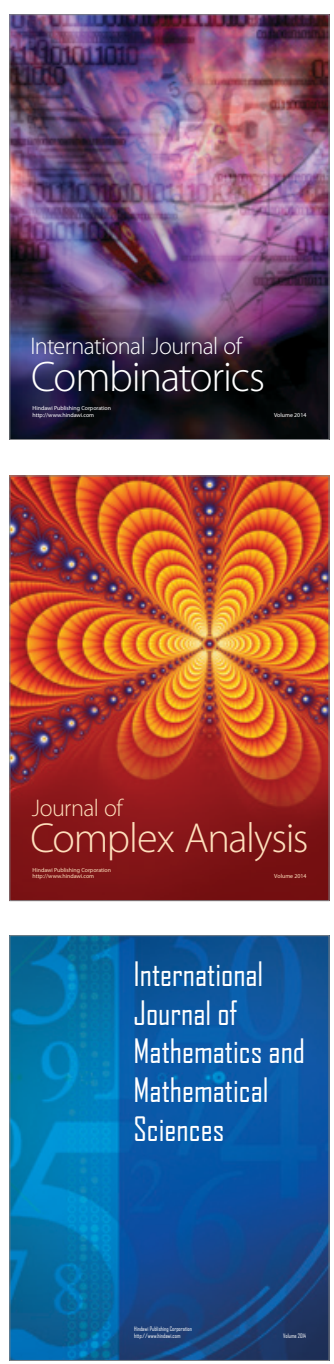
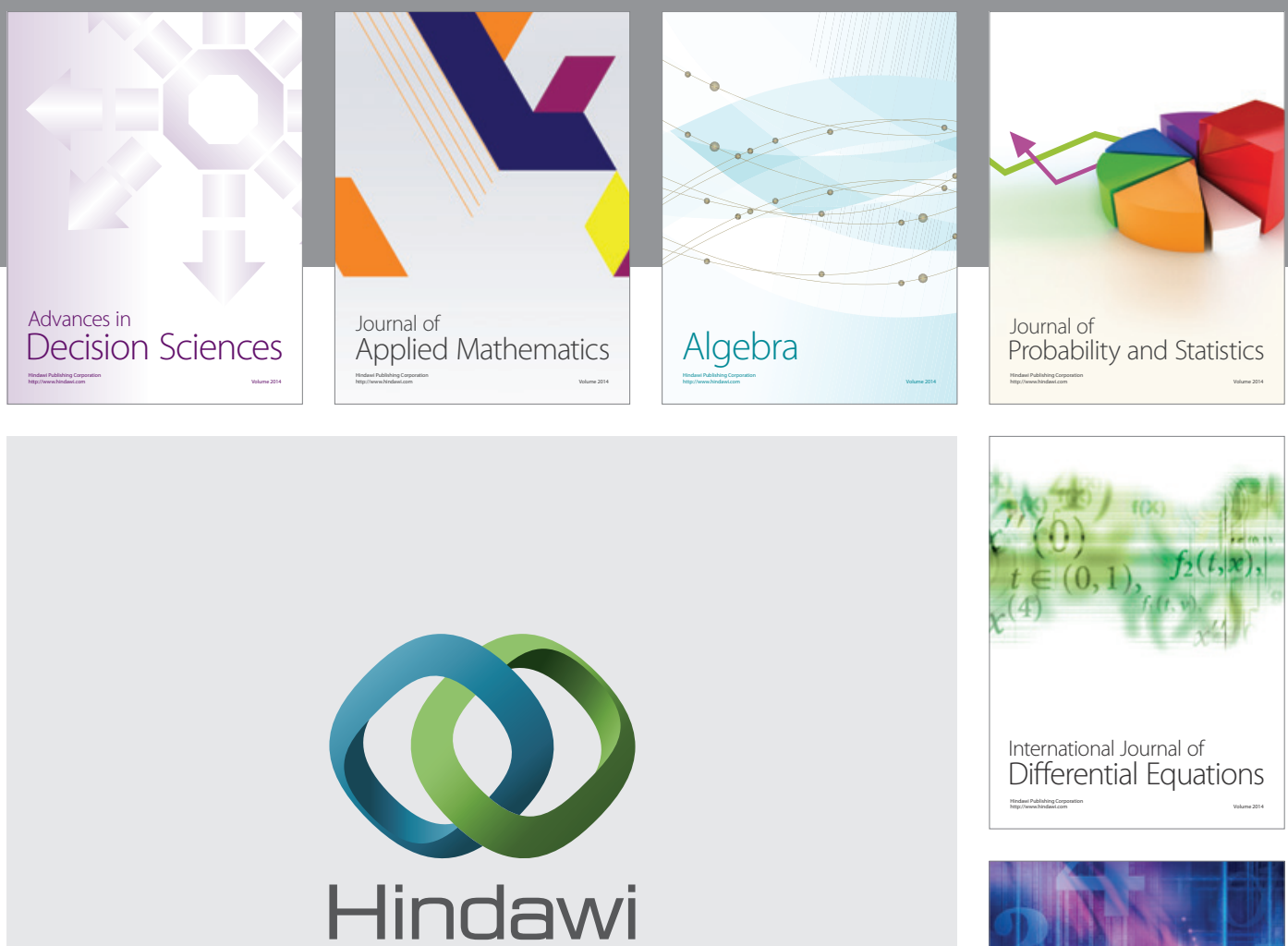

Submit your manuscripts at http://www.hindawi.com
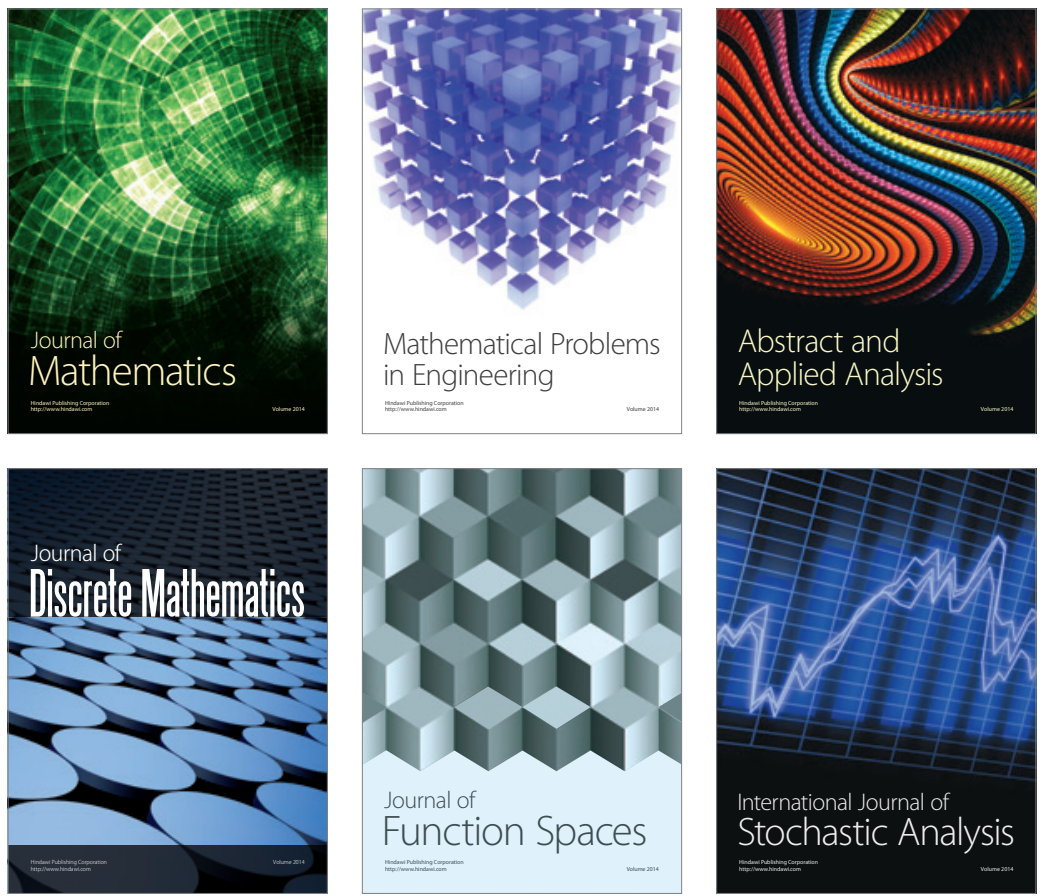

Journal of

Function Spaces

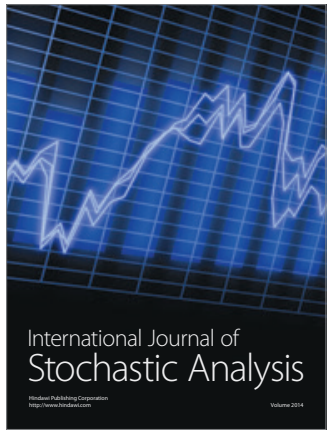

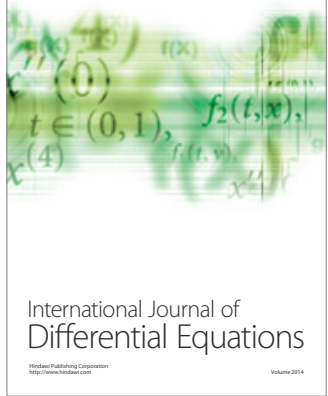
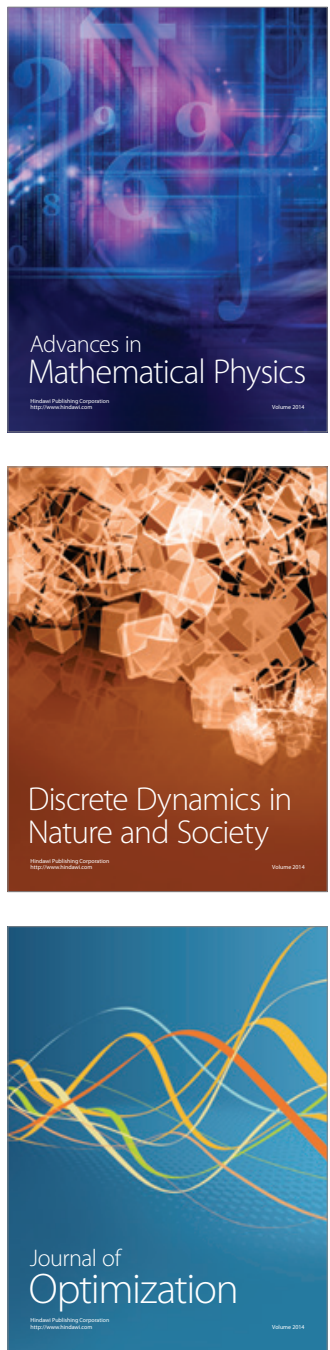